\title{
A Multidomain Model of Planar Electro-Active Polymer Actuators
}

\author{
Christoph M. Hackl, Hong-Yue Tang, Student Member, IEEE, Robert D. Lorenz, Fellow, IEEE, Lih-Sheng Turng, \\ and Dierk Schröder, Fellow, IEEE
}

\begin{abstract}
Dielectric electro-active polymers (EAPs) have a significant deformation response to electrical stimulation. The ease of fabrication of these materials makes them very suitable for product-integrated actuators. Existing electromechanical models do not fully integrate all aspects of the physical behavior needed to design such actuator systems. This paper presents a fully integrated, multidomain model for such EAP actuators, combining the electrostatic properties of the electrical circuit with the compliant electrodes, the actual force output in the translation direction, nonlinear elastic behavior, and damping of the polymer material. The capabilities of the developed model are discussed and verified with laboratory experiments.
\end{abstract}

Index Terms-Actuators, electro-active polymer (EAP) actuators, polymer actuators.

\section{INTRODUCTION}

D IELECTRIC electro-active polymers (EAPs) are lightweight, inexpensive, fracture tolerant, pliable, easily configurable, and have a significant deformation response to electrical stimulation [1]. The attractive characteristics offer the potential of these materials being suitable for product-integrated actuators, e.g., active damping actuators (ships, bridges...) or artificial muscles (far future). Current models [1]-[7], [10] do not fully integrate all of the physical domains needed to design actuator systems. A model, which fully integrates the multiple physical domains, but retains the electrostatic, mechanical, and mechanical models would substantially facilitate design.

This paper presents such a multiphysics model for EAP actuators, combining the electrostatic properties of the electrical

Paper IPCSD-05-039, presented at the 2004 Industry Applications Society Annual Meeting, Seattle, WA, October 3-7, and approved for publication in the IEEE TRANSACTIONS ON INDUSTRY APPLICATIONS by the Electric Machines Committee of the IEEE Industry Applications Society. Manuscript submitted for review November 19, 2004 and released for publication June 7, 2005. This work was supported by the Wisconsin Electric Machines and Power Electronics Consortium (WEMPEC) and the Polymer Engineering Center, both at the University of Wisconsin, Madison.

C. M. Hackl and D. Schröder are with the Department of Electrical Drive Systems, Technical University of Munich, 80333 Munich, Germany (e-mail: christoph.hackl@tum.de; dierk.schroeder@tum.de).

H.-Y. Tang is with the Department of Mechanical Engineering and Department of Electrical and Computer Engineering, University of Wisconsin, Madison, WI 53706 USA, and also with the Mechanical and Aeronautical Engineering Department, University of California, Davis, CA 95616 USA (e-mail: tangh@ucdavis.edu).

R. D. Lorenz is with the the Department of Mechanical Engineering and Department of Electrical and Computer Engineering, University of Wisconsin, Madison, WI 53706 USA (e-mail: lorenz@engr.wisc.edu).

L.-S. Turng is with the Department of Mechanical Engineering, University of Wisconsin, Madison, WI 53706 USA (e-mail: turng@engr.wisc.edu).

Digital Object Identifier 10.1109/TIA.2005.853384

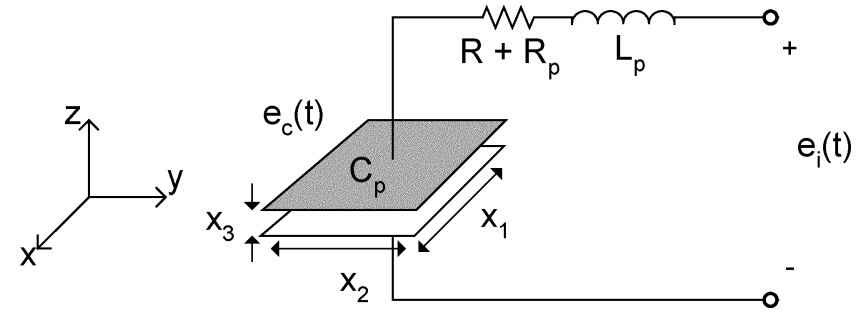

Fig. 1. Electrical circuit of polymer with compliant electrodes.

circuit with the physical properties of compliant electrodes; of the polymer material. The capabilities of the developed model are discussed and verified with laboratory experiments on test EAP actuators.

\section{NONLINEAR MODEL OF POLYMER ACTUATOR}

\section{A. Electrical Circuit of the Actuator}

The pre-strained polymer with applied compliant electrodes [1] on the top and bottom sides can be considered as a capacitance $C_{p}\left(x_{1}, x_{2}, x_{3}\right)$ depending on its geometry. Furthermore, the compliant electrodes and the special high-voltage cables can be treated as a serial connection of resistances $R_{p}+R$ and an inductance $L_{p}$. The electrical circuit is depicted in Fig. 1.

With Kirchhoff's Voltage Law the circuit can be described by

$$
e_{i}(t)=L_{p} \frac{d i}{d t}+\left(R_{p}+R\right) i(t)+e_{c}(t)
$$

Substituting

$$
e_{c}(t)=\frac{1}{C_{p}\left(x_{1}, x_{2}, x_{3}\right)} \int i(t) d t=\frac{1}{C_{p}\left(x_{1}, x_{2}, x_{3}\right)} q(t)
$$

and defining the system state vector as

$$
x(t)=\left(\begin{array}{l}
i \\
q
\end{array}\right)
$$

the state-space equations can be given by

$$
\frac{d i}{d t}=\frac{1}{L p}\left(-\left(R_{p}+R\right) i-\frac{1}{C_{p}\left(x_{1}, x_{2}, x_{3}\right)} q+e_{i}\right)
$$

and

$$
\frac{d q}{d t}=i
$$

The capacitance $C_{p}\left(x_{1}, x_{2}, x_{3}\right)$ will change when the dimensions (length $x_{1}$, width $x_{2}$, thickness $x_{3}$ ) of the polymer alter 


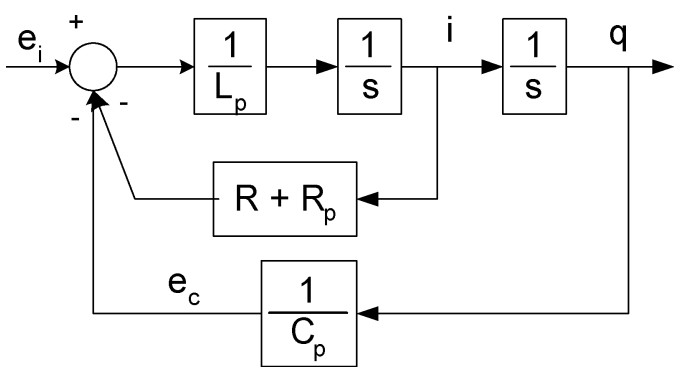

Fig. 2. State block diagram for the electrical circuit of the EAP actuator.

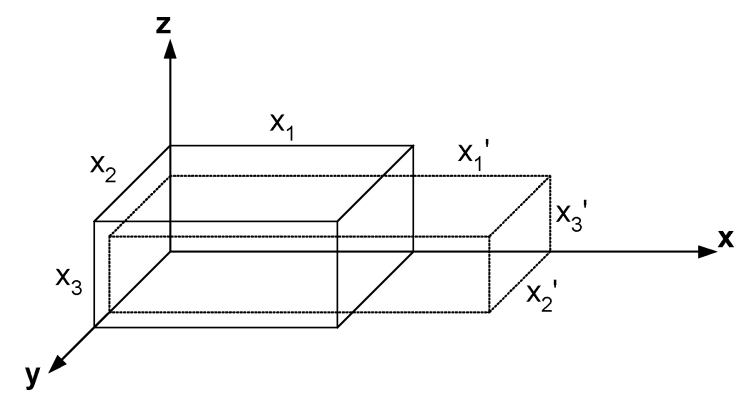

Fig. 3. General model of an incompressible polymer under stress.

by an external load. Fig. 2 shows how the electrical circuit can be transformed into a state block diagram.

Following the definition of $\lambda_{i}=x_{i}^{\prime} / x_{i}$ in Fig. 3, where $x_{i}$ is the original length and the prime symbol denotes the deformation length, with the assumption of incompressibility of the polymer material [1]-[4], [10] gives the relationship

$$
\lambda_{1} \lambda_{2} \lambda_{3}=1 \text {. }
$$

If one constrains $\lambda_{2}=1$ by the configuration of the actuator, the resulting deformation relationship becomes

$$
\lambda_{1}=\frac{1}{\lambda_{3}}=\lambda \text {. }
$$

Assuming that the polymer is the dielectric with the parallel plates formed in the $x-y$ plane, then $C_{p}$ can be computed by

$$
C_{p}(\lambda)=\frac{\varepsilon \varepsilon_{0} A}{d}=\frac{\varepsilon \varepsilon_{0} \lambda_{1} x_{1} \lambda_{2} x_{2}}{\lambda_{3} x_{3}}=\frac{\varepsilon \varepsilon_{0} \lambda^{2} x_{1} x_{2}}{x_{3}}
$$

which is a function of $\lambda$.

\section{B. Induced Pressure and Traction on the Actuator}

When charging the compliant electrodes, the unlike charges attract each other and induce a compressive pressure $\sigma_{\text {maxwell }}$ perpendicular to the area of the compliant electrodes [5], [6], [10]

$$
\sigma_{\text {maxwell }}=-\varepsilon \varepsilon_{0}\left(\frac{V}{x_{3}}\right)^{2} .
$$

Given that $q=C_{p} V=C_{p} e_{c}$, and (8) the physical compressive pressure can be restated as

$$
\sigma_{\text {maxwell }}=-\frac{1}{\varepsilon \varepsilon_{0}} \frac{1}{\lambda^{4}}\left(\frac{1}{x_{1} x_{2}}\right)^{2} q^{2} .
$$

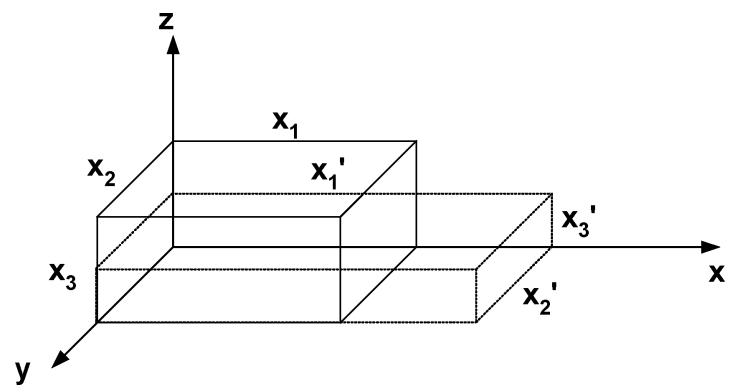

Fig. 4. The geometric model of an EAP actuator with $\lambda_{2}=1$.

This keeps the form consistent with the state variables selected in (2). The accumulation of charge on the capacitor plates will produce a pressure force. With the plates being the compliant electrodes, the electrostatic pressure will force the polymer actuator to mechanically contract in thickness and expand in area as depicted in Fig. 4.

\section{Dynamics and the Neo-Hookean Model of an EAP Actuator}

When subjected to modest loads, most solids obey Hookes Law, which is a linear elastic model. This model quickly fails when the strain becomes large. In the case of EAP material, the elastomer can deform as much as $800 \%$ in laboratory conditions. One of the features of the elastomers is that they can undergo large deformation from which they fully (or almost fully) recover, thus, no permanent deformation. There exists standard models such as Neo-Hookean and the Mooney-Rivlin [1], [2] model which utilize the strain energy function (SEF) and/or finite strain (SF) theories and a convolution relationship between stress and strain rate [1], [2]. The analysis of the actuator is then transformed into a plane-stress analysis.

The configuration or deformation of the actuator will be depending on the balance of stresses. The external load stress can be defined by

$$
\sigma_{\text {load }}=\frac{F_{L}}{\lambda_{2} x_{2} \lambda_{3} x_{3}} \approx \frac{F_{L}}{x_{2} x_{3}}
$$

where $F_{L}$ is the external load force. The approximation is appropriate because of the EAP actuator design where the two end areas are fixed. Formulating the problem by putting the $\sigma_{\text {maxwell }}$ on the $x-y$ plane, and the external load stress $\sigma_{\text {load }}$ on the $y-z$ plane, one can obtain the problem in a plane-stress configuration shown in Fig. 5.

For simplicity with respect to the Mooney-Rivlin model, the Neo-Hookean model has been used for the analysis. Following the strain energy function (SEF) of the Neo-Hookean material [2]

$$
U=C_{1}\left(I_{1}-3\right)
$$

where $I_{1}=\lambda_{1}^{2}+\lambda_{2}^{2}+\lambda_{3}^{2}$ is strain invariant and $C_{1}$ is a material constant [2], using the standard solid mechanics analysis one can compute the stress on each surface.

Following the Tonti diagram in finite-element method to decompose this as a plane-stress problem, making use of the de- 


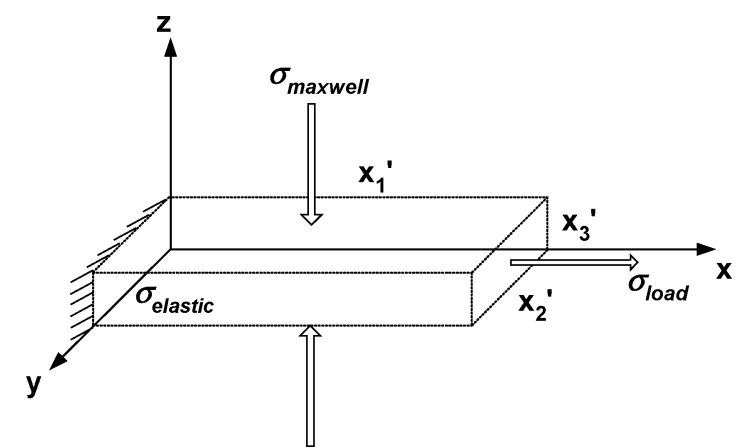

Fig. 5. Plane-stress formulation of the electroactive polymer actuator analysis whereby the left end is assumed to be fixed and the external load stress is acting along the $x$ direction and Maxwell (capacitor) pressure is acting in the $z$ direction.

formation relation $\lambda_{i}$, defining the configuration of the actuator $A$ as

$$
A=\left[\begin{array}{ccc}
\lambda_{1} & 0 & 0 \\
0 & \lambda_{2} & 0 \\
0 & 0 & \lambda_{3}
\end{array}\right]
$$

and formulating the nominal stress $S=J A^{-1} T$, where $J \equiv$ $\operatorname{det}(A)=1$ [2] by the incompressibility assumption of (5), and the stress matrix $T$ is defined by the conservation of linear momentum as

$$
T=\left[\begin{array}{ccc}
G \lambda_{1}^{2}-p & 0 & 0 \\
0 & G \lambda_{2}^{2}-p & 0 \\
0 & 0 & G \lambda_{3}^{2}-p
\end{array}\right]
$$

where $p$ is a hydrostatic force and $G$ is the shear modulus. Equating $S$ (by components) with the applied stress, yields

$$
\begin{aligned}
& \frac{1}{\lambda 1}\left(G \lambda_{1}^{2}-p\right)=\sigma_{\text {load }} \\
& \frac{1}{\lambda_{3}}\left(G \lambda_{3}^{2}-p\right)=\sigma_{\text {maxwell }}
\end{aligned}
$$

The relations suggest that the stresses are coupled by the incompressibility of the material. The deformation is also related to the material shear modulus, constant $G$.

It should be noted that even though the material can sustain large deformation, there is an operational limit at about $300 \%$ strain. Beyond $300 \%$ strain, an extremely large stress has to be applied to obtain further deformation. Following the kinetic theory of rubber elasticity [11], which is a special case of uniaxial loading under the Neo-Hookean model, the stress-strain relation can be expressed as

$$
\sigma=G_{0}\left(\lambda_{3}+\frac{1}{\lambda_{3}^{2}}\right)
$$

Fig. 6 shows this stress-strain relationship. The interpretation is that when a compression (negative) stress is applied, a substantial increases of stress is required beyond $\lambda_{3}=0.3$. In other words, when $\lambda_{3}<0.3$, a dramatic increase of $\sigma_{\text {maxwell }}$ is needed to give further extension in $\lambda_{1}$.

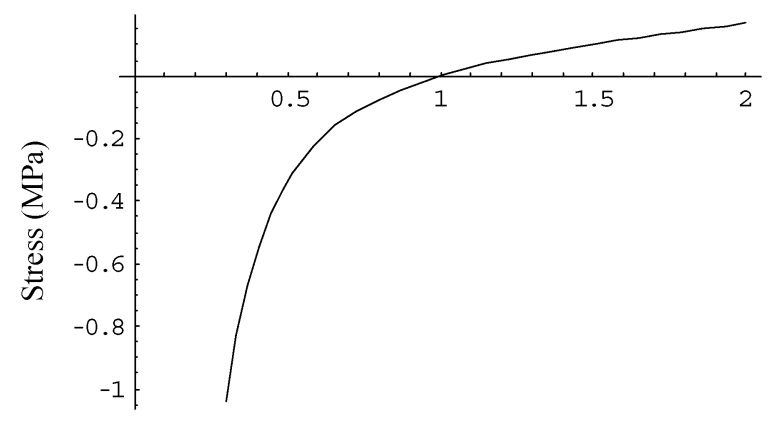

Extension Ratio $\left(\lambda_{3}\right)$

Fig. 6. Neo-Hookean model whereby for $\lambda_{3}<1$, a compression force is required and for $\lambda_{3}<0.3$, a dramatic increase of the stress suggests a theoretical limit to the maximum deformation.

\section{Combined Nonlinear Model and State Block Diagram}

Combining (9)-(12) and substituting in (6), one can arrive at the following stress balance relation which can be described by a function $h$ as shown in (14):

$h\left(q, F_{L}\right)=G\left(-\lambda^{3}+\frac{1}{\lambda}\right)+\lambda^{2} \frac{F_{L}}{x_{2} x_{3}}+\frac{1}{\varepsilon \varepsilon_{0}} \frac{1}{\lambda^{4}}\left(\frac{1}{x_{1} x_{2}}\right)^{2} q^{2}$

which relates the extension ratio $\lambda$, the charge $q$, and the external load force $F_{L}$. The material elasticity term

$$
\sigma_{\text {elastic }}=G\left(-\lambda^{3}+\frac{1}{\lambda}\right)
$$

remains in the equation. Thus, when $h\left(q, F_{L}\right)=0$, giving the balance between $\sigma_{\text {load }}, \sigma_{\text {elastic }}$, and $\sigma_{\text {maxwell }}$, there exists a real and positive $\lambda$ such that this equation holds. Therefore, at a given charge and external load, there exists a configuration such that the stresses are at equilibrium. A state block diagram can be used to reveal more insights.

Isolating $F_{L}$ in (14), adding a mechanical mass load $m$, with some assumed internal damping coefficient $b$, and rearranging the terms, one can arrive at an insightful force balance form which can be implemented in state block diagram format as shown in Fig. 7.

Note that this is not in the standard linear block diagram format since the terms are nonlinear and the cross-coupled feedbacks are shown as multi-input functional blocks. As suggested by (11) and (12), the stresses are coupled by the incompressibility, thus, the feedback of $\lambda$ can be seen in these blocks. The damping term $b$ is an inherent property of the polymer material.

A complete nonlinear state block diagram, which integrates the electrical and the mechanical model, is shown in Fig. 8. Noted that $C_{p}$ has been replaced by (7), and the output $x$ is the actual extension of the actuator. When a voltage is applied, charges are built up on the actuator causing the deformation. This model is now suitable for experimental evaluation and for integration into controls-oriented design of EAP actuator systems. 


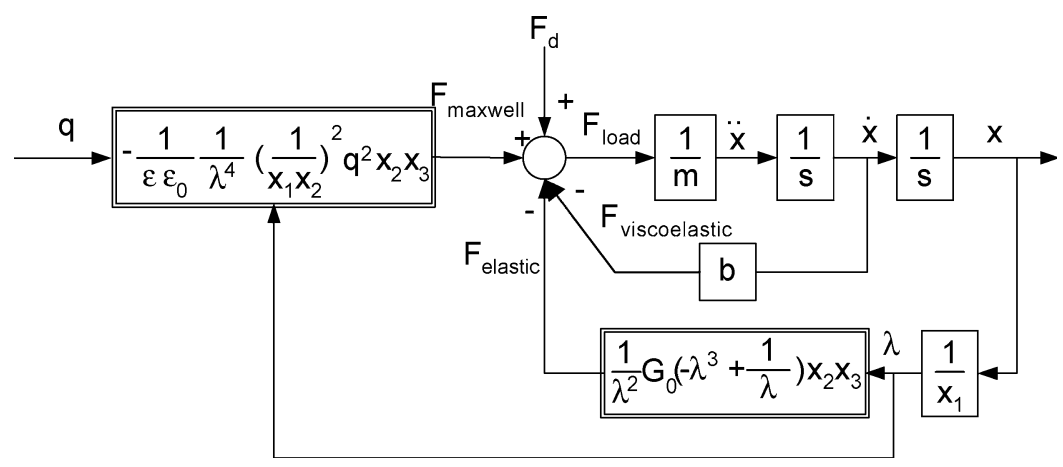

Fig. 7. Nonlinear state block diagram of the relation between the forces.

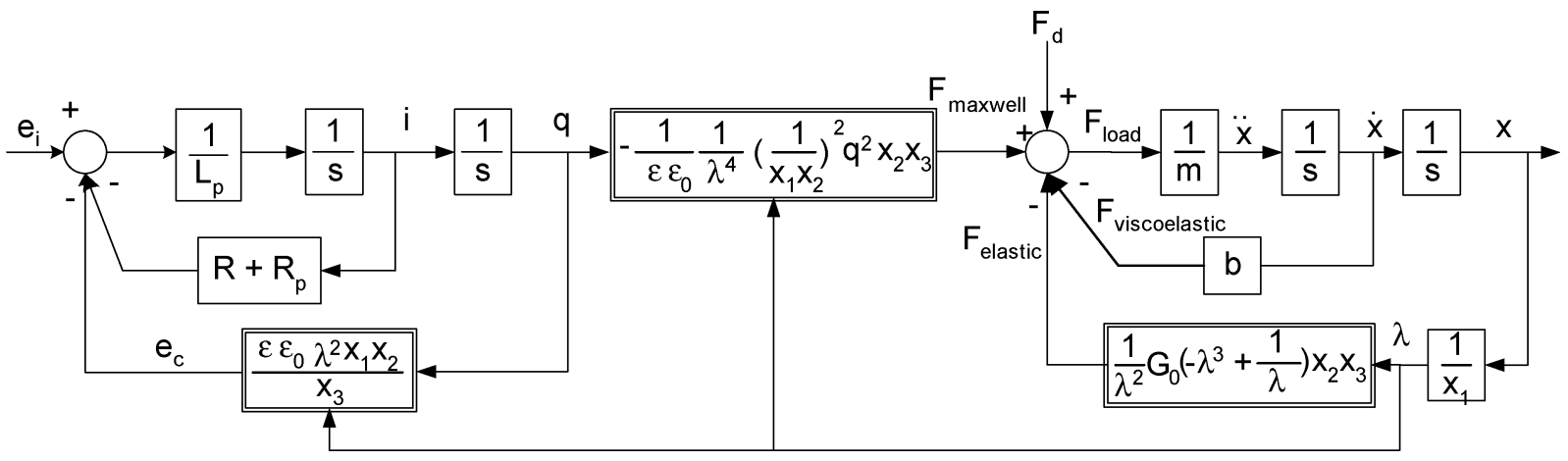

Fig. 8. Nonlinear state block diagram of the complete EAP actuator with an attached load mass.

\section{E. Command Feedforward (CFF) Realized by the Analytical Model}

CFF control can be used to improve command tracking, allowing the closed-loop controller to primarily be designed to improve disturbance rejection. CFF can be realized if the physical model of the system is known or well approximated. Since the electrical dynamics are much faster than the mechanical dynamics, the capacitor voltage can be well approximated by the supply voltage, from which one obtains a different form of the stress balance relationship (14) as

$$
h\left(V, F_{L}\right)=G\left(-\lambda^{3}+\frac{1}{\lambda}\right)+\lambda^{2} \frac{F_{L}}{x_{2} x_{3}}+\lambda^{2} \varepsilon \varepsilon_{0}\left(\frac{V}{x_{3}}\right)^{2} .
$$

Solving $h\left(V, F_{L}\right)$ for $V$ and rewriting as

$$
V=f\left(\lambda^{*}, F_{L}\right)=\frac{x_{3} \sqrt{-\frac{F \lambda^{* 2}}{x_{2} x_{3}}-G\left(\frac{1}{\lambda^{*}}-\lambda^{* 3}\right)}}{\lambda^{*} \sqrt{\varepsilon}}
$$

where $\lambda^{*}$ is the desired extension, one thus obtains $V$ as a function of $\lambda^{*}$ and $F_{L}$. Note that because of incompressibility, if $\lambda^{*}<\lambda$ where $\lambda$ is already determined by $F_{L}$ at the static state, then $V$ will be complex which implies that $\lambda^{*}$ is not achievable. Fig. 9 shows the nonlinear properties of the voltage, deformation, and load force relationship in a three-dimensional (3-D) format.

\section{PARAMETER ESTIMATION}

For observations and measurements, an EAP actuator was fabricated from 3M VHB 4905/4910 tapes with compliant electrodes made out of carbon black dust as shown in Fig. 10. The

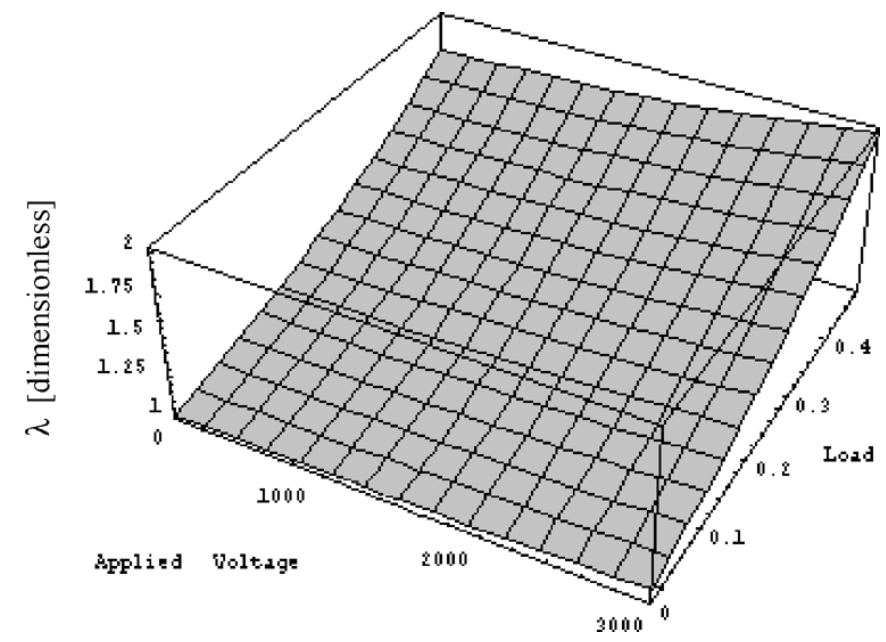

Fig. 9. Nonlinear relationship between the extension ratio $\lambda$, external load $F_{L}(\mathrm{~N})$, and the applied voltage $V(\mathrm{~V})$.

actuator is in the simplest single-sheet configuration with rigid mount in each end.

The testing apparatus consists of the test stage allowing translation motion, a host PC and a Hitachi H8/3664F digital signal processor (DSP) for interfacing the encoder, controlling the $\mathrm{dc}$ to high-voltage dc (DC/HVDC) converter, and other generalpurpose data collection tasks. The EMCO G30 DC-HVDC is a proportional amplifier converting 0-12 Vdc to 0-3000 Vdc. A Texas Instruments TI SN754410NE half-bridge circuit is used to drive the $\mathrm{dc} / \mathrm{HVdc}$ converter via a pulsewidth-modulation (PWM) scheme by the DSP. Cautions were taken to ensure instruments are isolated from the high voltage. 


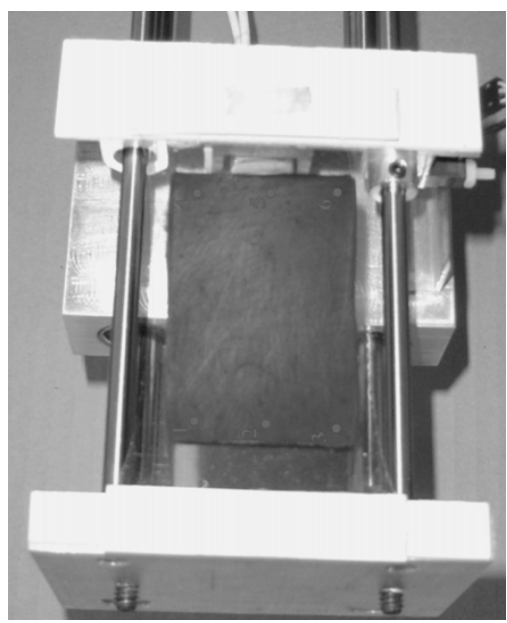

Fig. 10. Planar elastic polymer actuator in test stand.

TABLE I

PARAMETERS OF THE EXPERIMENTAL ACTUATOR

\begin{tabular}{c|c}
\hline Parameter & Value \\
\hline $\mathrm{R}_{\mathrm{p}}$ & $500 \mathrm{k} \Omega$ \\
\hline $\mathrm{R}$ (negligible) & $20 \mathrm{k} \Omega$ \\
\hline $\mathrm{L}_{\mathrm{p}}$ & $1.3 \mathrm{H}$ \\
\hline $\mathrm{M}$ & $70 \mathrm{~g}$ \\
\hline$\varepsilon_{r}$ & 4.8 \\
\hline $\mathrm{E}$ & $96 \mathrm{kN} / \mathrm{m}^{2}$ \\
\hline $\mathrm{x}_{1}$ & $35 \mathrm{~mm}$ \\
\hline $\mathrm{x}_{2}$ & $31 \mathrm{~mm}$ \\
\hline $\mathrm{x}_{3}$ & $0.5 \mathrm{~mm}$ \\
\hline
\end{tabular}

The parameters of the model were estimated or measured and are given in Table I.

\section{ANALYTICAL AND EXPERIMENTAL RESULTS}

The developed model was used to evaluate the design and behavior of the EAP actuator. The analytical results were compared with the experimental behavior of the actuator.

\section{A. Voltage-Displacement Relationship}

Fig. 11 overlays three voltage-displacement plots. The model results are similar to the experimental response. A fixed load is applied to the actuator to simulate a constant applied force.

The simulation (theoretical) model overestimates the response of the actuator due to the assumption of $\lambda_{2}=1$ is not fully satisfied by the crude fabrication of laboratory specimen. The growth rate of $\lambda$ should be reduced by $1 / \sqrt{2}$, which is shown as the corrected plot. Also, the data from the region near $2500 \mathrm{~V}$ are not accurate due to the noise from the dc to high-voltage dc converter. Thus, the data at that region do not truly reflect the behavior of the actuator.

\section{B. Deformation/Displacement}

The model estimated the actuator extension under various load and results were compared with the experiment data. A series of external load at $0.1-\mathrm{N}$ increment with $0.5-\mathrm{N}$ initial load

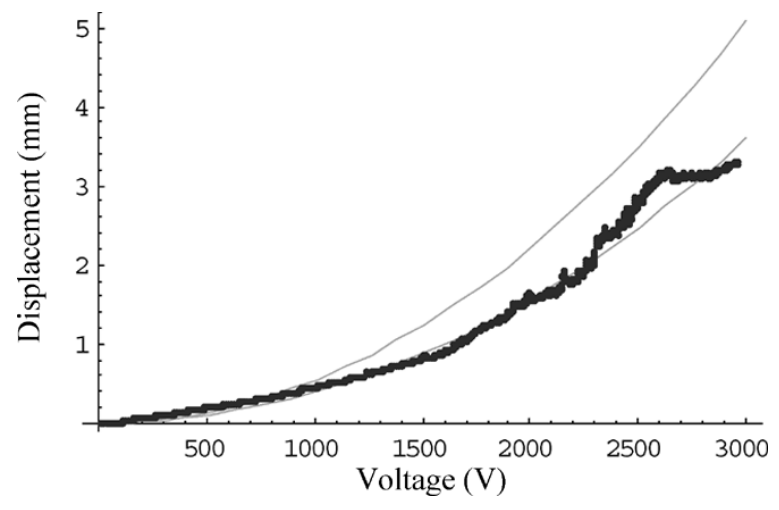

Fig. 11. Plot of the voltage versus displacement. Middle trace: experiment; upper trace: simulation; lower trace: simulation with correction factor.

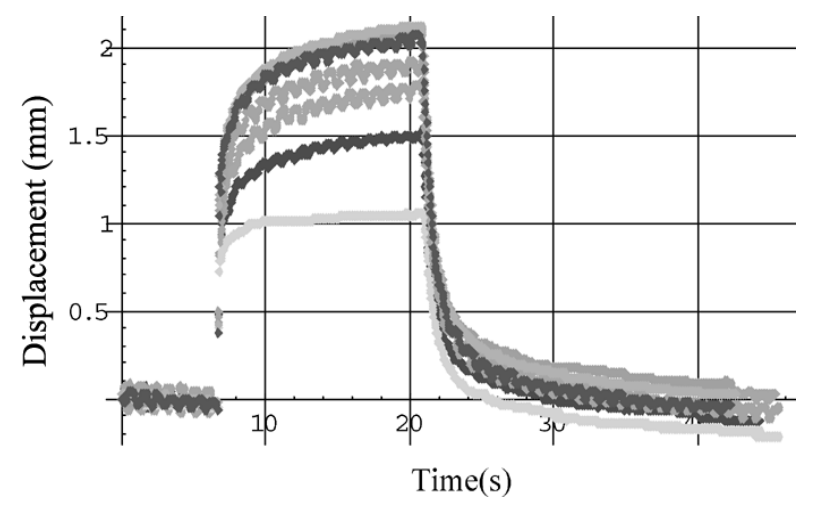

Fig. 12. Experimental results to a step input with external loads ranging from 0.5 to $1.1 \mathrm{~N}$.

from the static weight of the test stand were applied to the actuator, and a 3000-V step voltage was then applied and held for $12 \mathrm{~s}$ (applied at $7 \mathrm{~s}$ ). Fig. 12 shows the experimental results.

At constant voltage, small creep behavior has been observed. This relaxation phenomenon has been reported in [12]. The inaccuracy can be explained by the validity of the assumptions. With the increase of the external load causing the actuator to deform "out of shape," the geometry assumptions no longer hold. It could also due to the nonlinearity of the material and the accuracy of the Neo-Hookean model. The model begins to underestimate the extensions but it still can describe the general behavior up to the maximum strain limit.

\section{Nonlinear Polymer Behavior}

It is a known fact that viscoelastic materials often exhibit hysteresis. This can be observed during cyclic loading of the EAP material as shown in Fig. 13.

The developed model was then implemented in Matlab/Simulink and simulations were run with the introduced parameter values from the previous section. To include the effects of the viscoelasticity in the polymder material, a Kelvin viscoelastic model was included. The Kelvin viscoelastic model is a simple mechanical damper in parallel with a spring to augment the effect of the time-dependent behavior. An extensive list of model exists, such as the standard linear model shown in Fig. 14 which is used in finite-element tools such as FEMLab. 


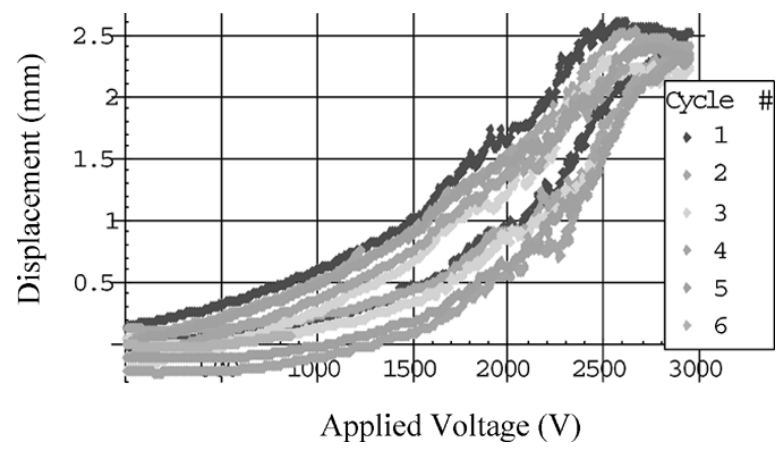

Fig. 13. Cyclic response of the EAP actuator.

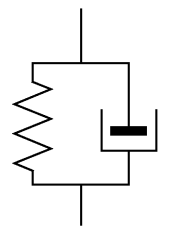

(a)

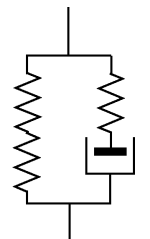

(b)
Fig. 14. (a) Kelvin model. (b) Standard linear model.

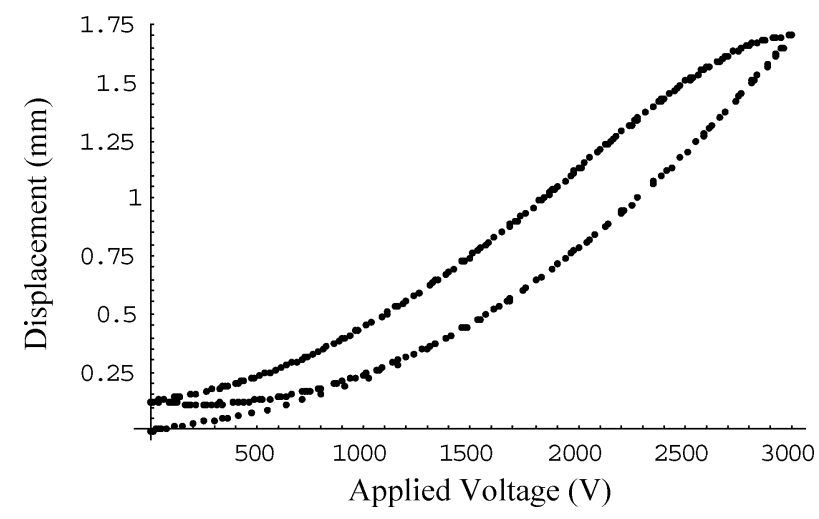

Fig. 15. Simulation of the response of the EAP actuator.

A few things are observed form the Matlab/Simulink simulation shown in Fig. 15. First is that the magnitude of the response is smaller. This is obvious by the fact that the Kelvin model reaches an intermediate state, thus, the absolute magnitude should be smaller. This also shows a limitation of using the Kelvin model in series with the analytical model.

\section{CONCLUSION}

The model presented integrates the multiphysics properties inherent in EAP actuators. It retains the physical attributes needed for design and modeling for control. It helps to understand the highly nonlinear behavior of EAP actuators stimulated by charges.

To properly describe the time-dependent behavior of the material used by the actuator, it is possible to model the system in finite-element tools such as FEMLab, and import it into Matlab/Simulink. The parameters required for the viscoelastic model can be obtain by static and dynamic mechanical evaluation. The preferred viscoelastic model will be a standard linear

model as it will have additional dynamics that are not included in the Kelvin model.

The experimental results also suggest some design and operational properties of EAP actuators. Viscoelasticity and other time-dependent properties such as creep are issues that are dependent on materials engineering.

\section{REFERENCES}

[1] Y. Bar-Cohen, Electroactive Polymer (EAP) Actuator As Artificial Muscles-Reality, Potential, and Challenges. Bellingham, WA: SPIE, 2001.

[2] G. A. Holzapfel, Nonlinear Solid Mechanics. Chichester, U.K.: Wiley, 2000.

[3] H. T. Banks and N. J. Lybeck, "Modeling methodology for elastomer dynamics," in Systems and Control in the Twenty-First Century, C. I Byrnes, B. N. Datta, D. S. Gilliam, and C. F. Martin, Eds. Boston, MA: Birkhäuser, Oct. 1996.

[4] H. T. Banks, N. J. Lybeck, M. J. Gaitens, B. C. Munoz, and L. C. Yanyo, "Modeling the dynamic mechanical behavior of elastomers," Center for Research in Scientific Computation, North Carolina State Univ., Raleigh, NC, Rep. CRSC-TR96-26, Oct. 1996.

[5] R. S. Rivlin, "Large elastic deformations of isotropic materials I, II, III," Phil. Trans. R. Soc. A, vol. 240, pp. 459-525, 1948.

[6] R. Pelrine, R. Kornbluh, Q. Pei, and J. Joseph, "High-speed electrically actuated elastomers with strain greater than 100\%," SRI International, Menlo Park, CA, Rep., 1999.

[7] R. Pelrine, R. Kornbluh, and G. Kofod, "High strain actuator materials based on dielectric elastomers," Adv. Mater., no. 12, pp. 1223-1225, 2000.

[8] Q. Wang, "Principle of electromechanical sensors and actuators," (ME 2082), Lecture, Dept. Mech. Eng., Univ. Pittsburgh, Pittsburgh, PA Spring 2003.

[9] "Nonlinear finite element analysis of elastomers," MSC Software Corp., Santa Ana, CA, Tech. Paper, 2000.

[10] C. M. Hackl, "A physics-based model of electro-active polymer actuators as the basis for a Gopinath-style motion state observer," Bachelors thesis, Lehrstuhl Elekt. Antriebssystem, Tech. Univ. München, Munich, Germany/ Dept. Mech. Eng. and Dept. Elect. Comput. Eng., Univ. Wisconsin, Madison, 2003.

[11] T. A. Osswald and G. M. Hanser, Materials Science of Polymers for Engineers, 2nd ed. Cincinnati, OH: Hanser Gardner, 2003, ch. 9.

[12] K.-C. Park, J. W. Youn, J. W. Jeon, H. Choi, H. Kim, and J.-D. Nam, "The analysis on the deformation of electrostrictive polymer film with respect to time under constant voltage," in Proc. Int. Conf. Control, Automation and Systems (ICCAS'01), 2001, pp. 1092-1097.

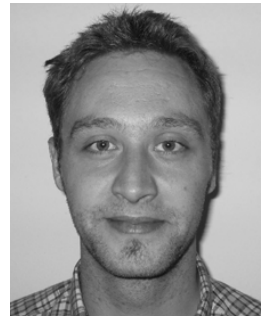

systems.

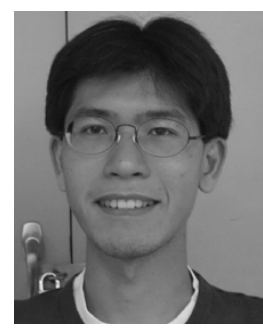

computer science.
Christoph M. Hackl was born in Mannheim, Germany, in 1977. He received the B.S.E.E degree from the Technical University of Munich (TUM), Munich, Germany, in co-operation with the University of Wisconsin, Madison, and the Diploma in Electrical Engineering from TUM in January and August 2004, respectively.

He is currently a Post-Graduate Associate at the Institute of Electrical Drive Systems, TUM. His main interests are robust, decentralized, and adaptive control strategies for uncertain and time-varying MIMO

Hong-Yue (Ray) Tang (S'05) was born in Hong Kong in 1980. He received the B.S. and M.S. degrees in mechanical engineering from the University of Wisconsin, Madison, in 2002 and 2004, respectively. $\mathrm{He}$ is currently working toward the Ph.D. degree at the University of California, Davis.

Since 2005, he has been a Teaching Assistant in the Mechanical and Aeronautical Engineering Department at the University of California, Davis. His main interests are in alternative energy, control and actuator technology, humanoid robotics, and 


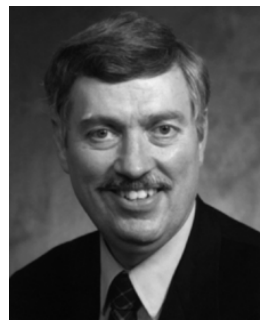

Robert D. Lorenz (S'83-M'84-SM'91-F'98) received the B.S., M.S., and Ph.D. degrees from the University of Wisconsin, Madison, and the M.B.A. degree from the University of Rochester, Rochester, NY.

Since 1984, he has been a member of the faculty of the University of Wisconsin, Madison, where he is the Mead Witter Foundation Consolidated Papers Professor of Controls Engineering in both the Department of Mechanical Engineering and the Department of Electrical and Computer Engineering. He is Co-Director of the Wisconsin Electric Machines and Power Electronics Consortium, which celebrated its 20th anniversary in 2001. It is the largest industrial research consortium on motor drives in the world. He is also the thrust leader for control and sensor integration in the Center for Power Electronic Systems, an NSF Engineering Research Center (ERC) which is a joint ERC with Virgina Polytechnic Institute and State University, Rensselaer Polytechnic Institute, University of Puerto Rico-Mayaguez, and North Carolina A\&T. From 1972 to 1982, he was a member of the research staff at the Gleason Works, Rochester, NY, working principally on high-performance drives and synchronized motion control. He was a Visiting Research Professor in the Electrical Drives Group, Catholic University of Leuven, Leuven, Belgium, in the summer of 1989 and in the Power Electronics and Electrical Drives Institute, Technical University of Aachen, Aachen, Germany, in the summers of 1987, 1991, 1995, 1997, and 1999, where he also was the SEW Eurodrive Guest Professor from September 1, 2000 until July 7, 2001. In 1969-1970, he conducted Master thesis research in adaptive control of machine tools at the Technical University of Aachen. His current research interests include sensorless electromagnetic motor/actuator technologies, real-time signal processing and estimation techniques, precision multiaxis motion control, and ac/dc drive and high-precision machine control technologies. He has authored more than 170 published technical papers and is the holder of 23 patents, with three more pending.

Dr. Lorenz is the IEEE Division II Director for 2005/2006, was the IEEE Industry Applications Society (IAS) President for 2001, a Distinguished Lecturer of the IAS for 2000/2001, immediate past Chair of the IAS Awards Department, and past Chairman of the IAS Industrial Drives Committee, and is a member of the IAS Industrial Drives, Electric Machines, Industrial Power Converter, and Industrial Automation and Control Committees. He is also the current Chair of the Periodicals Committee for the IEEE Technical Activities Board. He is a member of the IEEE Sensor Council AdCom. He was awarded the 2003 IEEE IAS Outstanding Achievement award, which honors his outstanding contributions and technological developments in the application of electricity to industry. $\mathrm{He}$ has won 18 prize paper awards. He is a Member of the American Society of Mechanical Enginees, Instrument Society of America, and The International Society for Optical Engineers. He is a Registered Professional Engineer in the States of New York and Wisconsin.

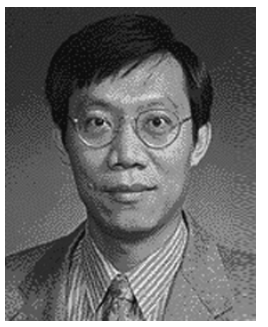

Lih-Sheng (Tom) Turng received the M.S. and $\mathrm{Ph} . \mathrm{D}$. degrees from Cornell University, Ithaca, NY, in 1987 and 1990, respectively.

$\mathrm{He}$ is currently an Associate Professor in the Department of Mechanical Engineering, University of Wisconsin, Madison (UW-Madison), where he is also the Co-Director of the Polymer Engineering Center, an NSF Multi-University Industry/University Cooperative Research Center (I/UCRC). He had been in industry for ten years before joining UW-Madison in 2000. His research interests are in the areas of injection molding, nanocomposites, biopolymers, modeling and simulation, and various innovative molding processes and materials. $\mathrm{He}$ is the Co-Editor of the Injection Molding Handbook (Cincinnati, OH: Hanser Gardner, 2002) and author of several books/book chapters and more than 100 papers.

Prof. Turng serves as the Chair of the Education Committee and the Board of Directors with the Injection Molding Division of the Society of Plastics Engineers (SPE) and is a Member of the Advisory Board for the Journal of Advances in Polymer Technology.

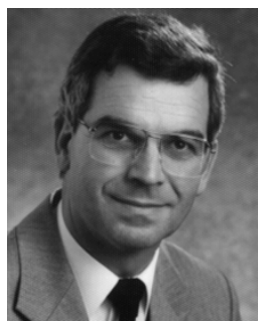

Dierk Schröder (M'84-SM'91-F'02) was born in Stendal, Germany, in 1941. He received the Dipl.-Ing. and Dr.-Ing. degrees from the Technical University of Darmstadt, Darmstadt, Germany, in 1966 and 1969, respectively.

He spent ten years in different positions with Asea, Brown Boveri (ABB). In 1979, he was named Professor and Chairman of the Institute of Electronics and Power Electronics at the University Kaiserslautern. In 1983, he was named Professor and Chairman of the Institute of Electrical Drive Systems at the Technical University of Munich, Munich, Germany. 